RESEARCH REPORT

\title{
Birth weight is inversely associated with coronary heart disease in post-menopausal women: findings from the British women's heart and health study
}

\author{
D A Lawlor, G Davey Smith, S Ebrahim
}

J Epidemiol Community Health 2004;58:120-125

See end of article for authors' affiliations

Correspondence to:

Correspondence to:
Dr D A Lawlor, Department of Social Medicine, University of Bristol

Canynge Hall, Whiteladies Road, Bristol BS8 2PR, UK; d.a.lawlor@bristol.ac.uk

Accepted for publication 2 September 2003

\begin{abstract}
Objectives: (1) To assess the association between birth weight and coronary heart disease (CHD) risk in a cohort of post-menopausal women, (2) to determine the combined effects of birth weight and adult body mass index on CHD, (3) to assess the role of insulin resistance as a mediating factor in the associations. Design: Cross sectional survey.

Setting: 23 British towns.

Participants: 1394 women aged 60-79 years.

Main outcome measures: coronary heart disease ( $n=199$ cases).

Results: Birth weight was inversely associated with CHD: age and survivor status of participant's mother adjusted odds ratio (95\% confidence intervals) per 1 standard deviation $(0.80 \mathrm{~kg})$ increase in birth weight was 0.84 (0.72 to 0.97$)$. This association strengthened to 0.80 (0.68 to 0.93 ) with further adjustment for adult body mass index, but there was no evidence of an interaction between birth weight and adult body mass index $(p=0.61)$. The association was not confounded by childhood or adulthood socioeconomic position or by adult smoking status of the participant. Adjustment for components of the insulin resistance syndrome attenuated the association to $0.87(0.72$ to 1.03$)$.

Conclusions: Intrauterine exposures that affect fetal growth also affect future adult CHD risk. The inverse association between birth weight and CHD may in part be mediated via insulin resistance.
\end{abstract}

B irth weight seems to be inversely associated with coronary heart disease (CHD) risk factors, such as hypertension, dyslipidaemia, and glucose intolerance. ${ }^{12}$ However, only a small number of studies have looked at the association between birth weight and CHD. Most, ${ }^{3-9}$ though not all ${ }^{10}{ }^{11}$ of these studies, have shown an inverse association between birth size and CHD in women and men, though results in women have often been imprecise because of the small number of CHD cases among women in several studies. ${ }^{12}$

The existence of a causal association between birth size and adult cardiovascular disease has been questioned, and in particular it has been suggested that socioeconomic position in childhood as well as adulthood may confound this association. ${ }^{13}$ In addition, with respect to the association of birth weight with blood pressure, which has been extensively investigated, the inverse association in many studies has been dependent upon adjustment for contemporary body mass index. ${ }^{13}{ }^{14}$ This finding has lead to the suggestion that post-natal factors that affect growth may be more important than intrauterine factors. ${ }^{14}$ To this end Lucas et al have proposed a series of four regression models to assess the individual and combined effects of birth weight and body mass index, in order to assess the fetal origins compared with post-natal hypotheses. ${ }^{14}$ In one study an interaction between birth weight and adult body mass index was found, such that the inverse association between birth weight and CHD was only present among men in the highest third of adult body mass index. ${ }^{6}$ In a second study men with CHD, compared with those without, had low birth weight and low weight at l year, followed by accelerated post-natal increase in weight and body mass index and, to a lesser extent, height. ${ }^{9}$ However, in most studies the inverse association between birth weight and CHD is not dependent upon adjustment for body mass index. ${ }^{48}$ To date, studies assessing the association of birth weight with CHD have not systematically applied the regression models suggested by Lucas et al. ${ }^{14}$

Insulin resistance may be an important mediator in the association between birth weight and CHD, either because of programming of changes in insulin metabolism and action ${ }^{15}$ or via genetic factors with pleiotropic effects on both insulin mediated fetal growth and insulin resistance in later life. ${ }^{16}$ However, no studies have specifically assessed the role of insulin resistance as a mediating factor in the birth weightCHD association. As ovarian function may be programmed during the intrauterine period, life time exposure to endogenous oestrogen may also mediate any association between birth weight and CHD in women. This could be assessed to some extent by examining the effect on any association of adjustment for menopausal age, though again to date this has not been investigated.

The aims of this study are to assess the association between birth weight and CHD risk in a cohort of post-menopausal women, to determine whether this is confounded by socioeconomic position in childhood and adulthood, to examine the combined effects of birth weight and adult body mass index on CHD, and to assess the role of insulin resistance and menopausal age as mediating factors in the association.

\section{METHODS}

\section{Participants}

This is a cross sectional examination of baseline data collected for the British women's heart and health study. Full details of the selection of participants and measurements have been previously reported. ${ }^{17}{ }^{18}$ Women aged $60-79$ years

Abbreviations: CHD, coronary heart disease; HOMA, homeostasis model assessment 


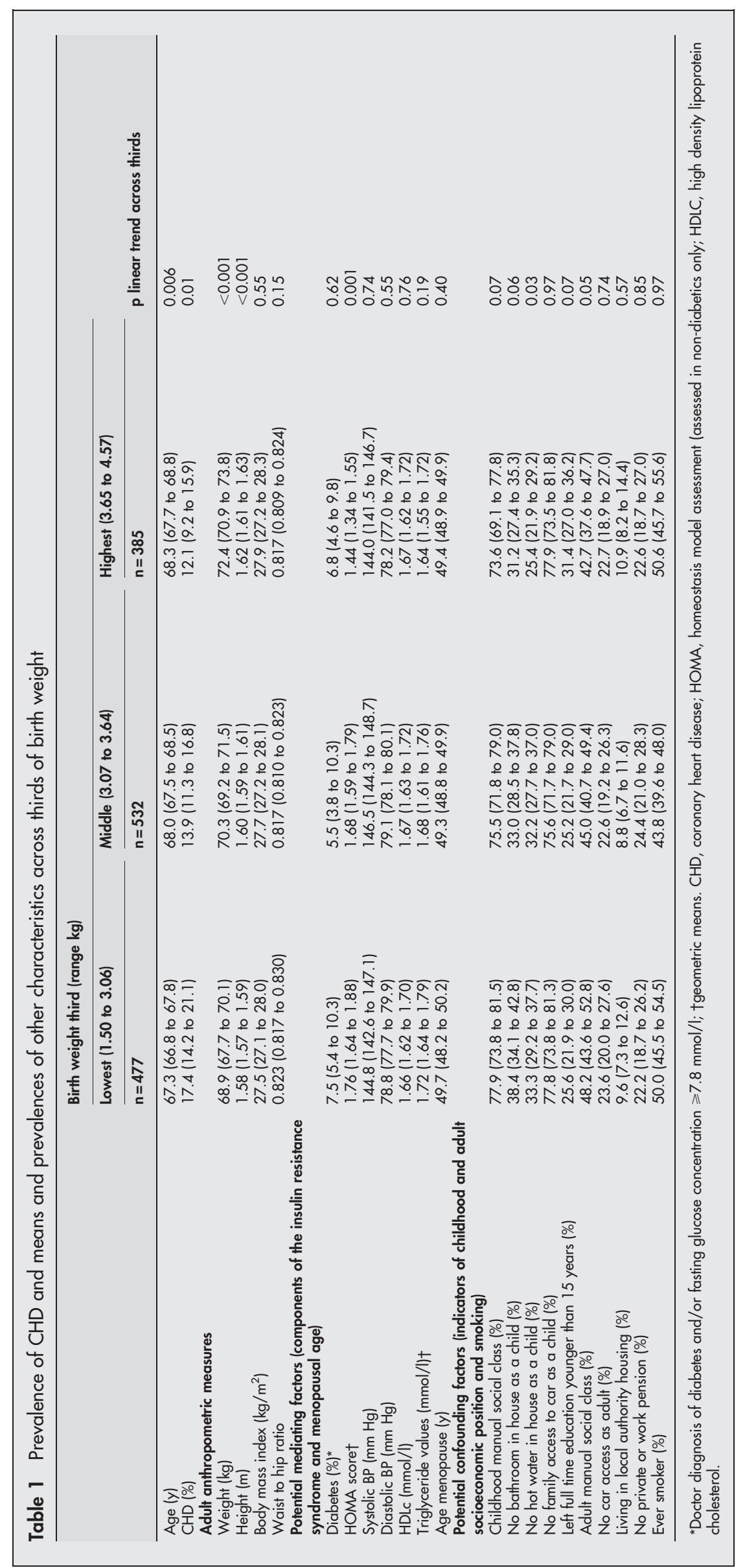




\section{Key points}

- Birth weight is inversely associated with coronary heart disease

- This association is not dependent upon adjustment for contemporary body size and is not confounded by socioeconomic position

- If maternal smoking during pregnancy is associated with offspring coronary heart disease then this may confound the association

- The association is in part mediated via insulin resistance

were randomly selected from general practitioner lists in 23 British towns. A total of 4286 women $(60 \%$ of the invited 7173) participated and baseline data (self completed questionnaire, research nurse interview, physical examination, and primary care medical record review) were collected between April 1999 and March 2001. Participants were slightly younger than non-responders but the prevalence of medical records of CHD were the same in participants and non-responders. ${ }^{17}$ Local ethics committee approvals were obtained.

\section{Birth weight}

The women were asked to report their birth weight in pounds (lb) and ounces (oz) in the self completed questionnaire. For comparisons with other studies the self reported birth weights were converted from $\mathrm{lb}$ to $\mathrm{kg}$ by multiplying by a factor of 0.4545 . Of the 4286 participants, 1394 (33\%) provided details of their birth weight. The analyses presented in this paper are restricted to those women with birthweight data.

\section{Coronary heart disease}

Prevalent CHD was defined as any participant with a primary care medical record (obtained from a review of the written notes, computer notes, and hospital discharge letters) of myocardial infarction or angina (including those with a medical record of having had an angioplasty or coronary artery bypass), or any participant who reported ever being diagnosed by a doctor with one of these conditions or having one of these operative procedures. ${ }^{17}$ Eighty per cent of those defined as having CHD were identified in both their medical records and from self report, 13\% were identified in their medical records only, and $7 \%$ by self report only. When sensitivity analyses were conducted including only those women with a medical record of $\mathrm{CHD}$ as a case the results did not differ from those presented here.

\section{Other variables}

Blood samples were taken after a minimum six hour fast (except for insulin dependent diabetics) using evacuated tubes. Serum insulin was measured using an enzyme linked immunosorbent assay (ELISA) assay that does not cross react with proinsulin. ${ }^{19}$ Insulin resistance was estimated according to the homeostasis model assessment (HOMA) as the product of fasting glucose $(\mathrm{mmol} / \mathrm{l})$ and insulin $(\mu \mathrm{U} / \mathrm{ml})$ divided by the constant 22.5. ${ }^{20}$ HOMA scores were not calculated for women with a fasting glucose of $\geqslant 7.8 \mathrm{mmol} / \mathrm{l}$ or those with a diagnosis of diabetes as the results are inaccurate in these groups. ${ }^{20}$ In total $98(7 \%)$ women of the 1394 included in the analysis had a diagnosis of diabetes and/or a fasting glucose level $\geqslant 7.8 \mathrm{mmol} / \mathrm{l}$; for the purpose of this analysis these women were all defined as having diabetes.

A Dinamap 1846SX vital signs monitor was used to measure blood pressure. Arm circumference was measured and the appropriate cuff size was used. The mean of two seated and resting measures was used in all analyses. Standing height was assessed using a Harpenden statiometer that measured to the nearest millimetre. Weight was measured in light clothing without shoes to the nearest $0.1 \mathrm{~kg}$ using Soenhle portable scales. Waist measurements were taken using the midpoint between the lowest rib and iliac crest and hip measurements using the largest circumference below the waist. A flexible metal tape was used and the mean of two measurements taken to the nearest millimetre was used in the analyses.

Adult social class was defined on the basis of the longest held occupation of her husband for married women and her own longest held occupation for single women, childhood social class was defined on the basis of the longest held occupation of the participants' father, and both were classified according to the registrar general's classification. ${ }^{21}$ Additional assessment of socioeconomic position in childhood and adulthood included self reports of whether the house the woman had lived in for the longest period as a child had an internal bathroom and whether it had a hot water supply, whether the family had access to a car when she was a child, the number of years of full time education completed by the participant, the participants current (adult) car access, housing tenure, and pension arrangements. Age at menopause was obtained from the self completed questionnaire in which women were asked to report in years the date of their last menstrual period. Women who had a hysterectomy before natural cessation of periods ( $\mathrm{n}=28$ with data on birth weight) were assigned the median ( 50 years) age of menopause for the remainder of the cohort.

\section{Statistical analysis}

\section{Validation of self reported birth weight}

A $\chi^{2}$ test for independence and Student's $t$ test were used to compare differences between women who reported their birth weight and those who did not. The validity of recalled birth weight was assessed by examining its association with adult anthropometric measures as in a previous study. ${ }^{6}$ As a further validation the distribution of birth weights among women in this study were compared with obstetric record recorded birth weights of British women of a similar age from two other studies: the Hertfordshire birth cohort (women born between 1923 and 1930) and the British 1946 birth cohort.

\section{Combined effects of birth weight and contemporary} size

The four models proposed by Lucas et a ${ }^{14}$ were fitted. These are: (a) the association between birth weight and CHD; (b) that between adult body mass index and CHD; (c) a model including both birth weight and adult body mass index, and (d) a model additionally including the interaction between birth weight and adult body mass index.

\section{Role of confounding and mediating factors in the association between birth weight and CHD}

Birth weights were divided into thirds (the slightly unequal sizes of these thirds are because participants tended to round their birth weights to the nearest pound). The nature and magnitude of the association between CHD and other characteristics with birth weight was assessed by presenting means and prevalences across these thirds of birth weight. Multiple linear regression was used to examine the 
Table 2 Association of birth weight with CHD with adjustment for potential confounding and mediating factors

\begin{tabular}{|c|c|c|c|}
\hline Model & $\begin{array}{l}\text { Number with } \\
\text { complete data }\end{array}$ & $\begin{array}{l}\text { Age and maternal survival } \\
\text { adjusted OR for CHD per } 1 \\
\text { SD }(0.80 \mathrm{~kg}) \text { increase in birth } \\
\text { weight }(95 \% \mathrm{Cl})\end{array}$ & $\begin{array}{l}\text { Fully adjusted OR for CHD } \\
\text { per } 1 \mathrm{SD}(0.80 \mathrm{~kg}) \text { increase } \\
\text { in birth weight }(95 \% \mathrm{Cl})\end{array}$ \\
\hline $\begin{array}{l}\text { Potential confounding factors } \\
\text { Indicators of childhood social } \\
\text { circumastances* }\end{array}$ & 1387 & $0.84(0.72$ to 0.97$)$ & $0.83(0.71$ to 0.96$)$ \\
\hline All confounding factorst & 1383 & $0.84(0.72$ to 0.97$)$ & $0.84(0.72$ to 0.97$)$ \\
\hline $\begin{array}{l}\text { Insulin resistance (HOMA } \\
\text { score)‡ }\end{array}$ & 1083 & $0.82(0.69$ to 0.98$)$ & 0.85 (0.72 to 1.01$)$ \\
\hline $\begin{array}{l}\text { Insulin resistance/diabetes } \\
\text { categories and other } \\
\text { components insulin resistance } \\
\text { syndrome§ }\end{array}$ & 1246 & $0.82(0.70$ to 0.97$)$ & $0.87(0.72$ to 1.03$)$ \\
\hline Menopausal age & 1342 & $0.83(0.72$ to 0.97$)$ & $0.83(0.71$ to 0.97$)$ \\
\hline \multicolumn{4}{|c|}{$\begin{array}{l}\text { *Indicators of childhood social circumstances: childhood occupational social class (entered as indicator variables: I, } \\
\text { II, IIInon-manual, IIImanual, IV, V), childhood house with bathroom, childhood house with hot water, family access } \\
\text { to car as a child (all binary variables); tall confounders: childhood and adulthood occupational social class (both } \\
\text { entered as indicator variables: I, II, Illnon-manual, Illmanual, IV, V, VI), childhood house with bathroom, childhood } \\
\text { house with hot water, family access to car as a child, car access as adult, living in local authority housing, no } \\
\text { private or work pension (all binary variables), age at leaving full time education (split into thirds and entered as } \\
\text { indicator variable) and smoking (never, past, and current-includes those who stopped in past six months, entered } \\
\text { as indicator variable); fanalysis among non-diabetics only; §components of insulin resistance syndrome: insulin } \\
\text { resistance/diabetes (quarters of HOMA score with fifth category= diabetes, entered as an indicator variable), } \\
\text { triglyceride concentrations (logged), waist to hip ratio (both entered as continuous variable), high density } \\
\text { lipoprotein cholesterol, systolic blood pressure, diastolic blood pressure (all categorised into quarters and entered } \\
\text { as indicator variables); Imenopausal age categorised into quarters and entered as indicator variable. }\end{array}$} \\
\hline
\end{tabular}

associations of birth weight with insulin resistance and components of the insulin resistance syndrome. Multiple logistic regression was used to examine the associations of birth weight with prevalent CHD, with adjustment for potential confounding factors and mediating factors. As HOMA scores are not calculated for subjects with diabetes, this measurement could not be entered into a regression model without exclusion of all individuals with diabetes. To enter full data on insulin resistance diabetes status in logistic regression models, a categorical variable combining both HOMA scores and diabetic status was created, with the first four categories defined by the quarters of the HOMA score and a fifth category containing individuals with diabetes.

HOMA scores and triglyceride values had positively skewed distributions but logged values were normally distributed; geometric means are presented and logged values were used in the regression models. With these transformations residuals were normally distributed in all models. Where HOMA scores and triglyceride values were considered as outcomes the regression coefficients were exponentiated and proportionate changes per change in birth weight presented. In all analyses robust confidence intervals were estimated which take into account the clustering between participants from the same towns.

\section{RESULTS}

\section{Validation of recalled birth weight}

Women who recalled their birth weight were younger (67.9 versus 69.4 years, $\mathrm{p}<0.001)$ and more likely to have a mother who was still alive $(10.9 \%$ versus $4.5 \%, \mathrm{p}<0.001)$ than those who did not recall their birth weights. However, the prevalence of $\mathrm{CHD}$ and the distributions of other characteristics of the women considered in the analyses presented here did not differ between those with and without birth weight data (all p values $>0.22$ ). The mean (standard deviation) of recalled birth weights was $3.28(0.80) \mathrm{kg}$, which is consistent with hospital records of women born between 1923 and 1930 in Hertfordshire $(3.42 \mathrm{~kg} \text {, SD not provided })^{4}$ and also with women participants in the 1946 British birth cohort (3.32 (0.49) kg (Dr D Kuh, University College London, personal communication). Table 1 shows the characteristics of women across thirds of birth weight. There was a graded positive association between recalled birth weight and both adult weight and adult height, as would be predicted from studies that have assessed the associations between observed birth weight and these adult anthropometric measures. ${ }^{22}$ There was a weak inverse linear association between birth weight and waist to hip ratio, which has also been demonstrated with recorded birth weight. ${ }^{23}$ Recalled birth weight was not strongly linearly associated with adult body mass index.

\section{Associations of birth weight and body mass index with CHD}

Of the 1394 women with birthweight data 199 had CHD, giving a prevalence $(95 \%$ CI) of $14.4 \%$ (12.6 to 16.4$)$. Because of the associations between age and having a mother who was still alive with ability to report birth weight all multivariable analyses have been adjusted for participant age and maternal survival status. There was an inverse association between birth weight and CHD prevalence without adjustment for body mass index: age and maternal survival status adjusted odds ratio of CHD for a one standard deviation increase in birth weight $(0.80 \mathrm{~kg})$ was $0.84(0.72$ to 0.97$), p=0.02$. There was a positive association between body mass index and CHD prevalence: age and maternal survival status adjusted odds ratio of CHD for a one standard deviation $\left(5.01 \mathrm{~kg} / \mathrm{m}^{2}\right)$ increase in body mass index was 1.25 ( 1.08 to 1.45$) \mathrm{p}=0.003$. When birth weight and body mass index were simultaneously included in the regression model the inverse association between birth weight and prevalence CHD strengthened to 0.80 (0.68 to $0.93) \mathrm{p}=0.004$ and the positive association between body mass index and CHD remained unchanged. There was no evidence of a statistical interaction between birth weight and body mass index in their association with prevalent CHD $(\mathrm{p}=0.61)$. 


\section{Effects of confounding and mediating factors on the associations between birth weight and CHD}

Table 1 shows the distributions of potential confounding and mediating factors across thirds of birth weight. Birth weight was inversely associated with insulin resistance (HOMA score) and triglyceride concentrations. For a one standard deviation increase in birth weight $(0.80 \mathrm{~kg})$ the HOMA score decreased by $8 \%$ (95\% CI: $4 \%$ to $13 \%), p<0.001$, with adjustment for age and maternal survival status and triglyceride levels decreased by $3 \%(0 \%$ to $5 \%), p=0.08$. Diabetes prevalence was lowest among those in the middle third of the birth weight distribution and there was no evidence of linear associations between birth weight and any of systolic or diastolic blood pressure or high density lipoprotein cholesterol. Birth weight was not strongly linearly associated with age at menopause. There were inverse associations between birth weight and belonging to a manual social class as a child, living in a house without a bathroom as a child, living in a house without hot water as a child, and belonging to a manual social class adulthood, but birth weight was not linearly associated with other indicators of socioeconomic position. Adult smoking status was not linearly associated with birth weight.

Table 2 shows the association between birth weight and CHD, with adjustment for potential confounding and mediating factors. There were small amounts of missing data on some potential confounding and mediating variables and HOMA scores were calculated only for women without diabetes. In this table age and maternal survival status adjusted odds ratios among only those with complete data on all variables included in the fully adjusted model are compared with the fully adjusted effect so that any change is not related to the fully adjusted analyses being performed on a subgroup. The age and maternal survival status adjusted odds ratios (third column) are all consistent with each other and with that for all 1394 women with birthweight data (0.84 $(0.72$ to 0.97$))$, suggesting that missing data have not lead to selection bias. In the fully adjusted models variables that were not linearly associated with birth weight (table 1) have been categorised and entered into models as indicator variables so that assumptions of linearity are not made. The inverse association between birth weight and CHD was not affected by adjustment for indicators of childhood socioeconomic position alone or by adjustment for all potential confounding factors (childhood and adult socioeconomic position and adult smoking status). To further assess the role of socioeconomic position as a confounding factor (or possible effect modifier) stratified analyses were performed with indicators of socioeconomic position that were strongly associated with birth weight. A variable "poor childhood housing" was derived, which indicated anyone who had lived in a house without a bathroom and/or hot water as a child. The age and maternal survivor status adjusted inverse association between birth weight and CHD was the same among both those who lived in poor childhood housing $(0.84$ (0.69 to 1.09)) and those who were not living in such circumstances $(0.82(0.67$ to 0.98$))$, p for heterogeneity $=0.40$. Similarly, the inverse association was the same among women from manual social classes in childhood $(0.82(0.70$ to 0.97$))$ compared with non-manual social classes $(0.87$ (0.61 to 1.28$)$ ), $\mathrm{p}$ for heterogeneity $=0.83$, and among those from manual social classes in adulthood $(0.82(0.68$ to 0.99$))$ and those from non-manual social classes $(0.84$ $(0.65$ to 1.08$)), \mathrm{p}$ for heterogeneity $=0.39$.

Adjustment for both insulin resistance alone (among nondiabetics) and for all components of the insulin resistance syndrome attenuated the association, though did not fully explain it. Adjustment for menopausal age did not affect the association between birth weight and CHD.

\section{DISCUSSION}

Birth weight was inversely associated with CHD, independently of childhood and adult social class and smoking status, in this cohort of post-menopausal women. The association between birth weight and CHD prevalence was not dependent upon adjustment for contemporary body mass index. These findings suggest that intrauterine exposures that affect fetal growth also affect CHD risk. Adjustment for components of the insulin resistance syndrome moderately attenuated the association, suggesting that insulin resistance may play a part as a mediator in this association. Age at menopause did not seem to be an important mediator in this association.

\section{Is the association attributable to confounding?}

Adverse childhood socioeconomic circumstances, independently of adult socioeconomic position, predict $\mathrm{CHD}^{24}$ and are associated with low birth weight. ${ }^{25}$ We have previously shown a strong and graded linear association between childhood socioeconomic position and CHD risk in this cohort of women. ${ }^{26}$ Here we have demonstrated that a number of childhood indicators of socioeconomic position (poor housing conditions and manual social class) are associated with low birth weight. Of the adult indicators of socioeconomic position only adult manual social class was linearly associated with birth weight. Clearly adult socioeconomic position cannot directly influence an individual's birth weight, but may act as a proxy for early life socioeconomic position. For this reason associations of early life socioeconomic position with birth weight are likely to be more robust than those with adult socioeconomic position, and complete control for the potential confounding effect of socioeconomic position in the association between birth weight and CHD will be best achieved by controlling for a number of indicators of socioeconomic circumstances from across the life course. Only one previous study has adjusted for socioeconomic position in both childhood and adulthood, and as with our study found that the association was not confounded by these factors. ${ }^{8}$ Our results together with this study suggest that the association is not attributable to confounding by socioeconomic position.

A second potential important confounder to consider is maternal smoking during pregnancy. Maternal smoking is strongly predictive of low birth weight and may affect offspring CHD risk as maternal smoking is associated with offspring lung function in adulthood, a risk factor for $\mathrm{CHD},{ }^{27}$ and because offspring of mothers who smoke may be more likely to smoke themselves. ${ }^{28}$ We adjusted for adult smoking status among participants and found that this did not confound the association. However, adjustment for maternal smoking status during pregnancy is likely to be more important. In particular, in this study we did not find that women who had ever smoked had lower birth weights than those who had never smoked. No other study has been able to adjust for maternal pregnancy smoking in the association between birth weight and CHD risk and if maternal smoking is associated with increased offspring CHD risk then this is a potentially important source of confounding in the association.

\section{Study limitations}

An important limitation was the use of self reported birth weight, and the fact that only one third of the original cohort had data on birth weight. Recalled birth weight was associated with adult anthropometric measures in the ways one would expect and the distribution of recalled birth weights in this cohort was similar to those of recorded weights for women in the Hertfordshire cohort and the 1946 British birth cohort. In a number of studies self reported birth weight has been found to correlate well with hospital records 
(correlation coefficients in the range of 0.64 to 0.86 ), though absolute levels of accuracy may be poor. ${ }^{29}$ High correlation coefficients between hospital recorded and self reported birth weights will mean that in analyses using self reported data, subjects will be ranked in a similar way to that which they would have been with hospital records, and linear associations should be only minimally diluted. Recall of birth weight in other studies, as in ours, is associated with age and whether the participants mother is still alive; in addition women are more likely to recall birth weight than are men. ${ }^{29}$ Participant age and the survival status of the participant's mothers are also likely to be associated with the participant's CHD risk. We therefore adjusted results for these characteristics.

We do not have data on gestational age of these women. Birth weight adjusted for gestational age in other cohorts has tended to be more strongly inversely associated with CHD than birth weight alone, ${ }^{89}$ and so our results may underestimate the association between intrauterine growth and CHD risk. As this analysis is cross sectional, survivor bias may be a problem. However, mortality from CHD before the age of 67 years (the mean age of women in this analysis) is rare and therefore survivor bias is unlikely to have importantly biased our results. Importantly, the magnitude of the association between birth weight and CHD found in our study is consistent with that found in two large prospective cohort studies with nearly $100 \%$ follow up and use of hospital recorded birth weights. ${ }^{89}$ A further limitation is the use of a single measure of fasting insulin and glucose for the assessment of insulin resistance. As the secretion of insulin is pulsatile this may have resulted in some inaccuracy of our assessment of insulin resistance and resulted in incomplete adjustment for its effect in the association. Thus the role of insulin resistance may be greater than suggested by these results.

\section{Conclusion}

We conclude that birth weight is inversely associated with CHD, that this association is not attributable to confounding by socioeconomic position in childhood or adulthood, and that insulin resistance may, at least in part, mediate the association. Several mechanisms have been proposed to explain this association, and all may be relevant. Adverse intrauterine environmental factors may "programme" permanent changes in organ development and metabolism (particularly those involved with insulin/glucose and lipid metabolism), leading to future adult disease. ${ }^{15}$ It has also been suggested that genetic polymorphisms with pleiotropic effects are associated both with insulin mediated fetal growth and insulin resistance, and thence CHD in later life. ${ }^{16}$ Although we found no interaction between birth weight and adult body mass index this finding does not preclude an important effect of accelerated post-natal growth in addition to intrauterine effects, as shown with data from the Helsinki cohort in which detail infant and childhood anthropometric measures are available. ${ }^{9}$ Future research should try to elucidate the pathophysiology underlying in this association.

\section{ACKNOWLEDGEMENTS}

The British women's heart and health study is co-directed by Professor Shah Ebrahim, Professor Peter Whincup, Dr Goya Wannamethee, and Dr Debbie A Lawlor. We thank Carol Bedford, Alison Emerton, Nicola Frecknall, Karen Jones, Rita Patel, Mark Taylor, and Katherine Wornell for collecting and entering data, all of the general practitioners and their staff who have supported data collection and the women who have participated in the study.

\section{Contributors}

All authors developed the study aim and design. DAL undertook the initial analysis and coordinated writing of the paper. All authors contributed to the final version. DAL acts as guarantor for the paper.

\section{Authors' affiliations}

D A Lawlor, G Davey Smith, S Ebrahim, Department of Social Medicine, University of Bristol, Bristol, UK

Funding: the British women's heart and health study is funded by the Department of Health. D A Lawlor was funded by a Medical Research Council/Department of Health training fellowship when this work was undertaken and is now funded by a Department of Health career scientist award. The views expressed in this publication are those of the authors and not necessarily those of the Department of Health or MRC.

Conflict of interests: none declared.

\section{REFERENCES}

1 Huxley RR, Shiell AW, Law CM. The role of size at birth and postnatal catchup growth in determining systolic blood pressure: a systematic review of the literature. J Hypertens 2000;18:815-31.

2 McKeigue PM, Lithell HO, Leon DA. Glucose tolerance and resistance to insulin-stimulated glucose uptake in mean aged 70 years in relation to size at birth. Diabetologia 1998;41:1133-8.

3 Barker DJ, Winter PD, Osmond C, et al. Weight in infancy and death from ischaemic heart disease. Lancet 1989; ii: 577-80.

4 Osmond C, Barker DJ, Winter PD, et al. Early growth and death from cardiovascular disease in women. BMJ 1993;307:1519-24.

5 Stein $\mathrm{CE}$, Fall $\mathrm{CH}$, Kumaran $\mathrm{K}$, et al. Fetal growth and coronary heart disease in south India. Lancet 1996;348:1269-73.

6 Frankel S, Elwood P, Sweetnam P, et al. Birthweight, body-mass index in middle age, and incident coronary heart disease. Lancet 1996;348: 1478-80.

7 Rich-Edwards JW, Stampfer MJ, Manson JE, et al. Birth weight and risk of cardiovascular disease in a cohort of women followed up since 1976. BMJ 1997;315:396-400.

8 Leon DA, Lithell HO, Vagero D, et al. Reduced fetal growth rate and increased risk of death from ischaemic heart disease: cohort study of 15000 Swedish men and women born 1915-29. BMJ 1998:317:241-5.

9 Eriksson JG, Forsen T, Tuomilehto J, et al. Early growth and coronary heart disease in later life: longitudinal study. BMJ 2001;322:949-53.

10 Eriksson M, Tibblin G, Cnattingius S. Low birthweight and ischaemic heart disease. Lancet 1994:343:731.

11 Gunnarsdottir I, Birgisdottir BE, Thorsdottir I, et al. Size at birth and coronary artery disease in a population with high birth weight. Am J Clin Nutr 2002;76:1290-4

12 Lawlor DA, Ebrahim S, Davey Smith G. A lifecourse approach to coronary heart disease and stroke. In: Kuh D, Hardy R, eds. A life course approach to women's health. Oxford: Oxford University Press, 2002:86-120.

13 Huxley R, Neil A, Collins R. Unravelling the fetal origins hypothesis: is there really an inverse association between birthweight and subsequent blood pressure? Lancet 2002;360:659-65.

14 Lucas A, Fewtrell MS, Cole TJ. Fetal origins of adult disease-the hypothesis revisited. BMJ 1999;319:245-9.

15 Hales CN, Barker DJ. The thrifty phenotype hypothesis. Br Med Bull 2001;60:5-20.

16 Hattersley AT, Tooke JE. The fetal insulin hypothesis: an alternative explanation of the association of low birthweight with diabetes and vascular disease. Lancet 1999;353:1789-92.

17 Lawlor DA, Bedford C, Taylor M, et al. Geographical variation in cardiovascular disease, risk factors and their control in older women: British women's heart and health study. J Epidemiol Community Health 2003:57:134-40.

18 Lawlor DA, Davey Smith G, Ebrahim S. Birth weight of offspring and insulin resistance in late adulthood: cross sectional survey using data from the British women's heart and health study. BMJ 2002;325:359-62.

19 Andersen L, Dinesen B, Jorgensen PN, et al. Enzyme immunoassay for intact human insulin in serum or plasma. Clin Chem 1993:39:578-82.

20 Matthews DR, Hosker JP, Rudenski AS, et al. Homeostasis model assessment: insulin resistance and beta-cell function from fasting plasma glucose and insulin concentrations in man. Diabetologia 1985;28:412-19.

21 Office of Population Censuses and Surveys. Classification of occupations and coding index. London: HM Stationery Office, 1980.

22 Miller FJW, Billewicz WZ, Thomson AM. Growth from birth to adult life of 442 Newcastle upon Tyne children. Br J Prev Soc Med 1972;26:224-30.

23 Law CM, Barker DJ, Osmond C, et al. Early growth and abdominal fatness in adult life. J Epidemiol Community Health 1992:46:184-6.

24 Davey Smith G, Ben-Shlomo Y, Lynch J. Life course approaches to inequalities in coronary heart disease risk. In: Stansfeld S, Marmot M, eds. Stress and the heart. London: BMJ Books, 2002:20-49.

25 Kramer MS, Seguin L, Lydon J, et al. Socio-economic disparities in pregnancy outcome: why do the poor fare so poorly? Paediatr Perinat Epidemiol 2000; 14:194-210.

26 Lawlor DA, Davey Smith G, Ebrahim S. The association of childhood socioeconomic position with coronary heart disease risk in post-menopausal women: findings from the British women's heart and health study. Am J Public Health (in press).

27 Upton MN, Watt GC, Davey Smith G, et al. Permanent effects of maternal smoking on offsprings' lung function. Lancet 1998;352:453.

28 Kandel DB, Udry JR. Prenatal effects of maternal smoking on daughters' smoking: nicotine of testosterone exposure? Am J Public Health 1999;89:1377-83.

29 Allen DS, Ellison GT, dos Santos Silva I, et al. Determinants of the availability and accuracy of self-reported birth weight in middle-aged and elderly women. Am J Epidemiol 2002;155:379-84. 